\title{
ON SYSTEMATISATION OF ESTONIANS' BODY BUILD DATA
}

\author{
Helje KaArma ${ }^{1}$, Gudrun Veldre ${ }^{1}$, Litidia Saluste ${ }^{1}$, \\ Mart Lintsi ${ }^{1}$, JAAn KASMeL ${ }^{1}$, Ene-Margit Tit' ${ }^{1}$, \\ Raini Stamm ${ }^{1}$, Maie Toomsalu ${ }^{1}$, Andres Arend ${ }^{2}$ \\ ${ }^{1}$ Centre for Physical Anthropology, University of Tartu, Tartu, Estonia \\ ${ }^{2}$ Department of Anatomy, University of Tartu, Tartu, Estonia
}

\begin{abstract}
Based on research results of women's, men's and children's body build, we present the arithmetic means of adult Estonian men and women (aged 20-70 years) as the national norms. Classifications based on gender and age enable to somatotype the subjects according to the classes of the classification.
\end{abstract}

Keywords: body build; height-weight classification; anthropometry

\section{INTRODUCTION}

The Centre for Physical Anthropology at the University of Tartu has achieved success in studying the regularities of body build structure. It has been established that the anthropometric structure forms a highly-correlated system of individual characteristics where the leading characteristics are height and weight. Changes in the relations between height and weight cause systemic changes in all height, breadth and depth measurements, circumferences and body proportions. Changes in proportions in the general cohort as well as in the pure types - pycnics and leptosomes - are related to changes in the relation between height and weight. No other special groups of body build have been found; the same regularities are valid for the general cohort and the groups of pycnics and leptosomes. This has enabled us to create a unified classification for characterisation of body build, which consists of the following classes: small, medium, large, pycnomorphs, leptomorphs [1, 2, 8, 9] (see Fig. 1). In principle, 
the same regularities are valid for the samples of women, men and schoolchildren $[3,4,5,6,10,11,12,13,14]$.

\begin{tabular}{|l|l|c|c|c|}
\hline \multicolumn{4}{|c|}{ Weight classes } \\
\hline \multirow{3}{*}{$\begin{array}{l}\text { Height } \\
\text { classes }\end{array}$} & Short & Light & Medium & Heavy \\
\cline { 2 - 4 } & Medium & Small & \multicolumn{1}{c|}{$\begin{array}{c}\text { Pycno- } \\
\text { morphic }\end{array}$} \\
\cline { 2 - 5 } & Tall & $\begin{array}{c}\text { Lepto- } \\
\text { morphic }\end{array}$ & Medium & Large \\
\hline
\end{tabular}

Figure 1. Body build classes

Considering the above-mentioned regularities of body build structure and possibilities of differentiation between somatotypes by means of a heightweight classification, the aim was to establish national height and weight norms for the majority of the population of Estonia, and, based on arithmetic means and standard deviations, to establish separate SD height-weight classifications for all age groups of 20-70-year-old men and women. This might facilitate the assessment of peculiarities of body build in medical, health promotional and nutritional studies.

\section{METHODS}

The plan for the study was approved by the Ministry of Social Affairs, and it was financed by the National Institute for Health Development [7]. The first task was to collect representative material about 20-70-year-old Estonian men and women. For this, Estonia was divided into four regions - Tallinn, Tartu, East-Estonia and West-Estonia. The data (age, gender, height, weight) were collected by 50 family physicians. The criteria for choice were Estonian ethnicity, age $20-70$ years, generally good health. The subjects could not have chronic diseases or physical disabilities. The subjects' names and personal codes were not recorded. The study was conducted from 2003 to 2005. Statistical analysis of data was performed by Säde Koskel, MSc in Mathematical Statistics. The consultant was Professor Emeritus Ene-Margit Tiit. Data were collected on 4587 Estonian women and 4034 men, 8621 in all. 


\section{RESULTS}

As a result of statistical analysis, we present the arithmetic means of height and weight of 20-70-year-old Estonian men and women, which we can treat as national norms. Next, based on the means and standard deviations, the limits of height and weight were calculated for the classes of the height-weight classification of 20-70-year-old men and women for each year of age. Here, the height-weight classification was concretised - the classes of both pycnomorphs and leptomorphs were divided into three - pycnomorphs 1, 2, 3, and leptomorphs 1, 2, 3 (see Figure 2). These classifications can be used to systematise all the different body measurements in age groups.

\begin{tabular}{|c|c|c|c|c|}
\hline & & \multicolumn{3}{|c|}{ Weight classes } \\
\hline & & Light & Medium & Heavy \\
\hline & Short & 1. Small & 5. Pyknomorphic & II \\
\hline \multirow{2}{*}{$\begin{array}{l}\text { Height } \\
\text { classes }\end{array}$} & Medium & $\begin{array}{c}\text { 4. Leptomorphic } \\
\text { I }\end{array}$ & 2. Medium & III \\
\hline & Tall & II & III & 3. Large \\
\hline
\end{tabular}

Figure 2. Body build classes.

As a result of the study, mean national norms are presented for assessment of height and weight of 20-70-year-old men and women.

Height-weight classifications compiled according to gender and age make it possible to classify the whole cohort and to associate body build with medical, health promotional and nutritional data.

\section{REFERENCES}

1. Kaarma H. (1981) Multivariate statistical analysis of the women's anthropometric characteristics system. Tallinn: Valgus.

2. Kaarma H. (1995) Complex statistical characterisation of women's body measurements. Anthrop. Anz. 53, 239-244.

3. Kaarma H., Saluvere K., Saluste L., Koskel S. (1997) Application of a 5-class classification of height and weight to systematize anthropometric data of 16-year-old Tartu schoolgirls. Papers on Anthropology VII, 160-173. 
4. Kaarma H., Stamm R., Veldre G., Kasmel J. (2000) Possibilities for classification of anthropometric data of 16-18-year-old Tartu schoolgirls considering their age and constitutional peculiarities. Papers on Anthropology IX, 64-81.

5. Kaarma H., Kasmel J., Koskel S., Tiit E.-M. (2003) Changes in schoolgirls' body measurements during a 30-year period. Growth, Developments \& Aging, 67, 95-105.

6. Kaarma H., Stamm R., Kasmel J., Koskel S. (2005a) Body build classification for ordinary schoolgirls (aged 7-18 years) and volleyball girls (aged 13-16 years). Anthrop. Anz., 63, 77-92.

7. Kaarma H. (2005b) Final report of the agreement between the Centre for Physical Anthropology at the University of Tartu and the National Institute for Health Development. Unpublished document. Tartu.

8. Kaarma H. (2012) Twenty years of the Centre for Physical Anthropology. Papers on Anthropology XXII, 11-16.

9. Kaarma H., Saluste L., Lintsi M., Kasmel J., Veldre G., Tiit E.-M., Stamm R., Toomsalu M. (2016) Height and weight norms and somatotypical height-weight classification for 20-70-year-old Estonian Women. Human Evolution, 31, 4, 215-227.

10. Lintsi M., Kaarma H., Saluste L., Vasar V. (2002) Systemic changes in body structure of 17-18-year-old schoolboys. Homo 53, 157-169.

11. Lintsi M, Kaarma H. (2004) Body structure of eighteen-year-old conscripts assessed by anthropometry and dual-energy X-ray absorptiometry. Mankind Quart. 44, 3-28.

12. Maiste E., Kaarma H., Thetloff M. (1999) On the prospects of multivariate systematization of separate body measurements and indices of 15-year-old Estonian schoolgirls. Homo 50, 18-32.

13. Peterson J., Kaarma H., Koskel S. (2007) Using a height-weight classification for analysis of food energy and main nutrient content in 24-hour menus of 17-23-year-old Estonian female students. Anthrop. Anz. 65, 51-59.

14. Raschka, C. (2006) Sportanthropologie. Köln: Sportverlag Strauss.

\section{Address for correspondence:}

Helje Kaarma Dr. Sc., Dr. med.

Centre for Physical Anthropology

Institute of Anatomy

Faculty of Medicine

University of Tartu

E-mail: antrop@ut.ee 
Table 1. Mean values of height and weight for Estonian men aged $20-70$ years $(n=4034)$

\begin{tabular}{|c|c|c|c|c|c|c|c|c|c|}
\hline \multirow[b]{2}{*}{ Age } & \multirow[b]{2}{*}{$\mathbf{N}$} & \multicolumn{4}{|c|}{ Height } & \multicolumn{4}{|c|}{ Weight } \\
\hline & & Min & Max & Mean & SD & Min & Max & Mean & SD \\
\hline 20 & 123 & 161.00 & 198.00 & 180.85 & 7.56 & 51.00 & 107.00 & 75.15 & 10.83 \\
\hline 21 & 104 & 156.00 & 197.00 & 180.55 & 7.47 & 43.00 & 122.00 & 76.61 & 13.51 \\
\hline 22 & 105 & 165.00 & 203.00 & 181.54 & 7.99 & 56.90 & 123.70 & 78.54 & 13.24 \\
\hline 23 & 82 & 163.00 & 205.00 & 181.00 & 7.69 & 53.00 & 113.00 & 79.86 & 12.95 \\
\hline 24 & 107 & 160.00 & 196.00 & 180.79 & 6.44 & 57.00 & 120.00 & 77.67 & 11.41 \\
\hline 25 & 82 & 167.00 & 200.00 & 180.69 & 6.77 & 57.50 & 139.00 & 80.17 & 14.62 \\
\hline 26 & 80 & 163.00 & 198.60 & 180.94 & 7.23 & 59.00 & 149.70 & 82.84 & 16.84 \\
\hline 27 & 101 & 162.50 & 195.00 & 182.24 & 5.86 & 48.00 & 132.70 & 81.97 & 12.49 \\
\hline 28 & 97 & 163.00 & 194.10 & 180.00 & 6.52 & 50.70 & 128.00 & 83.78 & 15.08 \\
\hline 29 & 94 & 160.50 & 192.60 & 180.21 & 6.33 & 61.00 & 126.50 & 82.02 & 12.13 \\
\hline 30 & 79 & 166.60 & 197.00 & 180.67 & 6.31 & 56.00 & 120.00 & 83.92 & 13.07 \\
\hline 31 & 80 & 169.00 & 197.00 & 180.94 & 5.87 & 63.50 & 122.00 & 86.08 & 13.64 \\
\hline 32 & 101 & 165.00 & 196.00 & 180.34 & 5.68 & 60.00 & 115.80 & 83.09 & 11.96 \\
\hline 33 & 91 & 168.00 & 199.90 & 181.99 & 6.53 & 57.50 & 127.10 & 85.95 & 14.47 \\
\hline 34 & 116 & 164.00 & 200.00 & 179.40 & 6.36 & 63.00 & 132.00 & 83.52 & 14.35 \\
\hline 35 & 72 & 165.00 & 196.00 & 179.47 & 7.10 & 58.20 & 111.00 & 83.88 & 12.10 \\
\hline 36 & 86 & 158.00 & 203.00 & 181.03 & 6.64 & 50.00 & 145.00 & 87.83 & 16.02 \\
\hline 37 & 96 & 162.00 & 197.00 & 181.14 & 5.97 & 52.00 & 135.10 & 88.03 & 15.60 \\
\hline 38 & 90 & 165.00 & 196.30 & 180.04 & 6.29 & 60.00 & 151.00 & 88.14 & 18.07 \\
\hline 39 & 67 & 164.00 & 200.00 & 177.92 & 7.38 & 59.00 & 132.00 & 83.42 & 14.78 \\
\hline 40 & 79 & 158.30 & 200.00 & 179.27 & 7.12 & 63.00 & 123.00 & 87.45 & 14.82 \\
\hline 41 & 81 & 163.50 & 197.40 & 180.24 & 7.05 & 61.00 & 144.00 & 86.83 & 14.64 \\
\hline 42 & 98 & 164.00 & 196.00 & 178.99 & 7.04 & 60.00 & 120.00 & 86.19 & 14.58 \\
\hline 43 & 97 & 163.00 & 194.00 & 178.00 & 6.74 & 60.00 & 138.00 & 87.90 & 14.95 \\
\hline 44 & 87 & 164.00 & 193.00 & 179.25 & 6.07 & 65.00 & 130.00 & 88.09 & 14.31 \\
\hline 45 & 90 & 167.00 & 196.00 & 179.31 & 6.27 & 54.00 & 132.00 & 87.96 & 14.53 \\
\hline 46 & 83 & 162.00 & 200.00 & 180.00 & 7.00 & 65.00 & 140.00 & 91.77 & 14.88 \\
\hline 47 & 88 & 159.50 & 190.00 & 179.04 & 6.93 & 61.00 & 144.00 & 87.22 & 13.50 \\
\hline 48 & 84 & 165.00 & 194.20 & 177.50 & 6.65 & 60.00 & 127.00 & 85.37 & 13.60 \\
\hline 49 & 89 & 160.00 & 195.00 & 176.75 & 6.49 & 60.00 & 117.00 & 83.85 & 10.36 \\
\hline 50 & 77 & 155.00 & 193.50 & 178.15 & 7.00 & 49.90 & 142.90 & 87.51 & 14.32 \\
\hline 51 & 67 & 168.00 & 197.00 & 177.47 & 5.88 & 51.00 & 111.90 & 84.36 & 12.65 \\
\hline 52 & 98 & 157.00 & 192.00 & 177.52 & 6.30 & 57.40 & 115.00 & 86.86 & 11.86 \\
\hline 53 & 84 & 162.00 & 195.00 & 177.06 & 6.88 & 60.80 & 125.00 & 87.78 & 14.87 \\
\hline
\end{tabular}




\begin{tabular}{c|c|cccc|cccc}
\hline \multirow{2}{*}{ Age } & \multirow{2}{*}{$\mathbf{N}$} & \multicolumn{6}{|c}{ Height } & \multicolumn{4}{c}{ Weight } \\
\cline { 3 - 9 } & & Min & Max & Mean & SD & Min & Max & Mean & SD \\
\hline 54 & 81 & 160.00 & 193.00 & 177.79 & 6.28 & 55.00 & 138.00 & 86.47 & 14.62 \\
\hline 55 & 84 & 162.00 & 196.00 & 178.29 & 7.16 & 56.10 & 139.00 & 88.94 & 16.33 \\
\hline 56 & 80 & 160.00 & 193.50 & 176.79 & 6.67 & 54.00 & 130.00 & 85.08 & 14.04 \\
\hline 57 & 87 & 163.00 & 195.00 & 175.78 & 6.33 & 55.00 & 133.00 & 85.79 & 13.98 \\
\hline 58 & 76 & 161.00 & 190.00 & 176.19 & 6.19 & 54.40 & 118.00 & 86.67 & 13.93 \\
\hline 59 & 79 & 156.00 & 190.00 & 176.93 & 6.81 & 59.00 & 127.00 & 87.90 & 14.71 \\
\hline 60 & 48 & 158.00 & 188.00 & 175.55 & 8.07 & 57.00 & 140.00 & 87.29 & 17.25 \\
\hline 61 & 45 & 161.00 & 189.00 & 175.27 & 6.24 & 57.90 & 110.00 & 84.83 & 12.26 \\
\hline 62 & 48 & 156.00 & 197.00 & 174.88 & 7.46 & 54.00 & 136.00 & 83.14 & 15.11 \\
\hline 63 & 54 & 162.00 & 190.00 & 175.71 & 6.62 & 57.70 & 130.00 & 84.49 & 14.73 \\
\hline 64 & 52 & 158.00 & 190.00 & 176.36 & 6.47 & 67.00 & 126.00 & 89.93 & 13.65 \\
\hline 65 & 78 & 156.00 & 191.00 & 174.82 & 6.93 & 53.00 & 130.00 & 87.24 & 17.21 \\
\hline 66 & 35 & 162.00 & 184.00 & 172.25 & 5.76 & 60.00 & 112.30 & 83.82 & 14.04 \\
\hline 67 & 41 & 160.00 & 186.00 & 174.52 & 6.56 & 54.00 & 109.40 & 86.67 & 13.57 \\
\hline 68 & 31 & 165.00 & 188.00 & 176.05 & 6.22 & 58.40 & 109.00 & 82.64 & 12.84 \\
\hline 69 & 39 & 160.50 & 187.60 & 174.53 & 5.93 & 58.00 & 115.50 & 85.68 & 12.81 \\
\hline 70 & 21 & 157.00 & 181.00 & 172.87 & 5.59 & 72.00 & 124.00 & 89.15 & 12.90 \\
\hline & & & & & & & & &
\end{tabular}

Table 2. Mean values of height and weight for Estonian women aged 20-70 ( $n=4587)$

\begin{tabular}{c|c|cccc|cccc}
\hline \multirow{2}{*}{ Age } & \multirow{2}{*}{$\mathbf{N}$} & \multicolumn{6}{|c}{ Height } & \multicolumn{4}{c}{ Weight } \\
\cline { 3 - 9 } & & Min & Max & Mean & SD & Min & Max & Mean & SD \\
\hline 20 & 128 & 156.00 & 181.00 & 167.89 & 5.46 & 41.00 & 93.00 & 60.65 & 8.75 \\
\hline 21 & 107 & 152.70 & 186.40 & 167.49 & 5.95 & 41.60 & 86.40 & 59.54 & 8.07 \\
\hline 22 & 176 & 144.20 & 184.00 & 167.11 & 6.25 & 43.70 & 90.00 & 61.05 & 8.26 \\
\hline 23 & 113 & 146.00 & 181.00 & 167.35 & 6.39 & 38.00 & 101.00 & 60.82 & 9.95 \\
\hline 24 & 98 & 152.00 & 180.10 & 167.49 & 6.17 & 45.00 & 102.80 & 61.74 & 10.46 \\
\hline 25 & 94 & 149.40 & 185.00 & 166.85 & 6.71 & 43.30 & 90.50 & 61.09 & 10.03 \\
\hline 26 & 106 & 154.50 & 182.00 & 167.08 & 5.34 & 47.00 & 111.00 & 63.90 & 11.65 \\
\hline 27 & 119 & 148.00 & 184.00 & 166.95 & 6.05 & 40.00 & 118.00 & 62.70 & 11.50 \\
\hline 28 & 121 & 154.00 & 182.00 & 166.78 & 5.89 & 46.00 & 105.50 & 63.00 & 10.87 \\
\hline 29 & 115 & 152.30 & 185.00 & 166.89 & 6.70 & 40.00 & 95.00 & 63.74 & 11.17 \\
\hline 30 & 93 & 146.00 & 183.00 & 167.04 & 6.77 & 40.00 & 130.00 & 66.45 & 13.72 \\
\hline 31 & 91 & 152.00 & 178.00 & 166.96 & 5.28 & 45.30 & 130.00 & 67.17 & 14.53 \\
\hline 32 & 135 & 152.00 & 180.20 & 166.65 & 5.78 & 45.00 & 115.00 & 64.44 & 11.73 \\
\hline 33 & 82 & 152.00 & 182.00 & 166.48 & 5.50 & 45.00 & 119.00 & 66.56 & 13.74 \\
\hline 34 & 107 & 148.00 & 185.50 & 167.66 & 6.54 & 45.60 & 109.20 & 66.55 & 12.31 \\
\hline
\end{tabular}




\begin{tabular}{|c|c|c|c|c|c|c|c|c|c|}
\hline \multirow{2}{*}{ Age } & \multirow{2}{*}{$\mathbf{N}$} & \multicolumn{4}{|c|}{ Height } & \multicolumn{4}{|c|}{ Weight } \\
\hline & & Min & Max & Mean & SD & Min & Max & Mean & SD \\
\hline 35 & 107 & 151.00 & 178.50 & 166.59 & 6.44 & 44.40 & 113.00 & 67.22 & 12.37 \\
\hline 36 & 106 & 150.40 & 184.00 & 166.17 & 5.21 & 44.00 & 124.00 & 68.15 & 12.67 \\
\hline 37 & 103 & 150.40 & 183.00 & 166.75 & 6.28 & 46.00 & 109.00 & 68.00 & 12.78 \\
\hline 38 & 79 & 154.70 & 178.00 & 166.29 & 4.73 & 45.20 & 110.00 & 68.68 & 13.76 \\
\hline 39 & 98 & 150.00 & 180.00 & 166.16 & 6.05 & 44.00 & 117.00 & 67.82 & 13.17 \\
\hline 40 & 100 & 154.80 & 187.00 & 166.27 & 5.46 & 50.00 & 140.00 & 68.38 & 13.44 \\
\hline 41 & 93 & 147.60 & 176.00 & 166.08 & 5.91 & 42.80 & 114.00 & 70.24 & 13.29 \\
\hline 42 & 125 & 152.00 & 181.00 & 166.85 & 4.94 & 44.00 & 118.00 & 70.82 & 12.60 \\
\hline 43 & 102 & 151.80 & 189.00 & 165.27 & 5.74 & 48.00 & 112.00 & 70.90 & 14.18 \\
\hline 44 & 86 & 150.00 & 185.00 & 165.26 & 5.88 & 50.00 & 116.00 & 70.81 & 13.53 \\
\hline 45 & 109 & 150.50 & 189.80 & 165.02 & 6.67 & 44.00 & 117.00 & 73.13 & 14.81 \\
\hline 46 & 89 & 150.00 & 178.00 & 164.82 & 5.89 & 53.50 & 125.10 & 73.03 & 12.79 \\
\hline 47 & 111 & 153.00 & 180.00 & 165.66 & 5.07 & 51.00 & 106.00 & 69.48 & 10.19 \\
\hline 48 & 91 & 145.50 & 190.00 & 164.62 & 6.62 & 47.00 & 120.00 & 72.61 & 13.02 \\
\hline 49 & 88 & 138.00 & 182.00 & 163.01 & 6.47 & 45.00 & 130.00 & 74.02 & 16.70 \\
\hline 50 & 97 & 154.00 & 176.00 & 164.04 & 4.84 & 48.00 & 118.50 & 71.55 & 12.63 \\
\hline 51 & 79 & 151.00 & 182.00 & 164.15 & 5.26 & 45.00 & 109.80 & 71.44 & 13.02 \\
\hline 52 & 134 & 152.00 & 178.00 & 164.45 & 5.69 & 48.00 & 132.70 & 72.98 & 13.09 \\
\hline 53 & 76 & 150.00 & 180.00 & 163.95 & 5.47 & 46.80 & 105.00 & 74.90 & 12.78 \\
\hline 54 & 93 & 152.00 & 179.00 & 163.41 & 5.34 & 49.00 & 98.00 & 73.56 & 12.14 \\
\hline 55 & 93 & 152.00 & 178.00 & 163.61 & 5.21 & 49.00 & 125.00 & 73.61 & 14.71 \\
\hline 56 & 76 & 151.00 & 179.00 & 163.45 & 5.40 & 50.80 & 116.00 & 77.31 & 15.64 \\
\hline 57 & 96 & 147.50 & 175.00 & 163.69 & 5.27 & 51.70 & 130.70 & 77.17 & 15.70 \\
\hline 58 & 73 & 150.00 & 180.00 & 163.42 & 5.57 & 47.00 & 120.00 & 75.92 & 14.55 \\
\hline 59 & 82 & 152.90 & 180.00 & 162.51 & 5.48 & 48.90 & 135.50 & 75.27 & 14.82 \\
\hline 60 & 51 & 147.10 & 174.00 & 162.03 & 5.82 & 48.00 & 114.00 & 77.22 & 14.66 \\
\hline 61 & 35 & 150.00 & 177.00 & 162.10 & 6.48 & 46.00 & 125.00 & 78.23 & 16.10 \\
\hline 62 & 45 & 150.00 & 176.00 & 162.28 & 6.59 & 52.00 & 106.00 & 76.46 & 13.15 \\
\hline 63 & 46 & 149.10 & 174.50 & 161.92 & 5.32 & 41.00 & 114.50 & 73.83 & 15.59 \\
\hline 64 & 49 & 150.00 & 180.00 & 163.63 & 6.15 & 49.10 & 115.00 & 77.87 & 15.24 \\
\hline 65 & 71 & 150.00 & 173.00 & 161.74 & 5.62 & 46.00 & 128.70 & 73.71 & 15.64 \\
\hline 66 & 50 & 143.00 & 176.50 & 161.36 & 6.87 & 51.00 & 107.00 & 77.10 & 12.23 \\
\hline 67 & 39 & 156.50 & 174.70 & 162.80 & 4.25 & 48.50 & 102.00 & 74.69 & 14.08 \\
\hline 68 & 39 & 152.00 & 175.60 & 162.54 & 5.67 & 52.00 & 114.00 & 77.22 & 15.37 \\
\hline 69 & 52 & 150.00 & 171.00 & 161.37 & 4.94 & 50.00 & 110.00 & 73.80 & 13.50 \\
\hline 70 & 39 & 151.00 & 175.00 & 160.77 & 5.49 & 58.50 & 100.00 & 76.75 & 11.33 \\
\hline
\end{tabular}




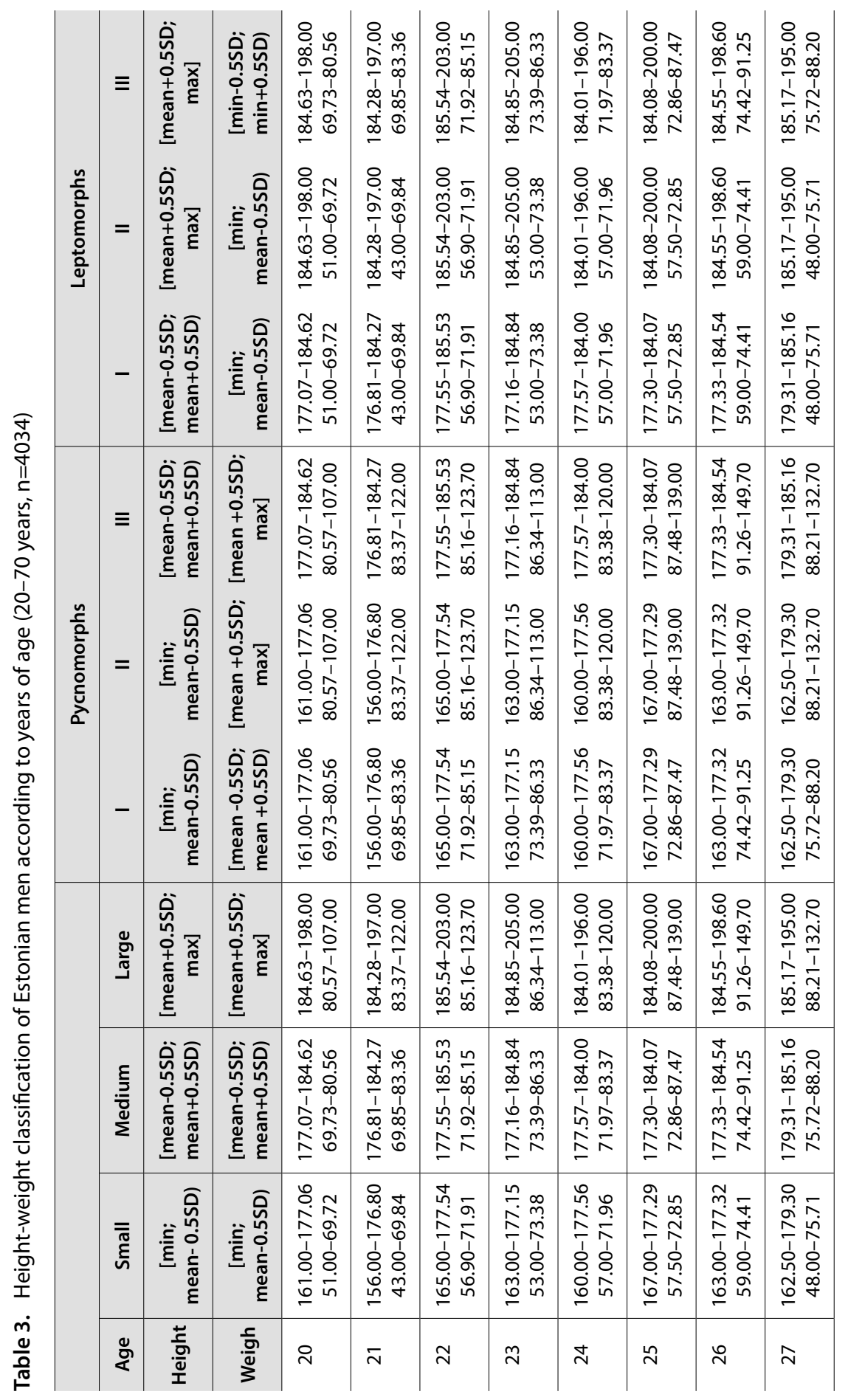




\begin{tabular}{|c|c|c|c|c|c|c|c|c|c|c|c|c|}
\hline 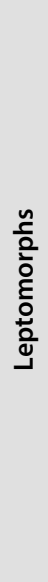 & $=$ & 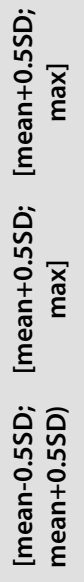 & 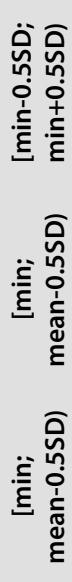 & 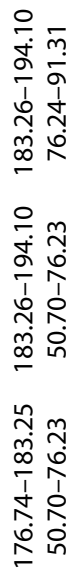 & 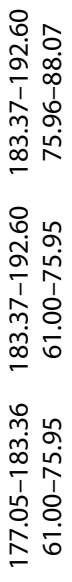 & 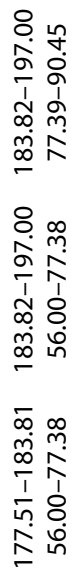 & 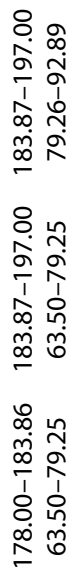 & 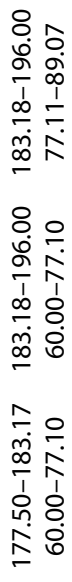 & 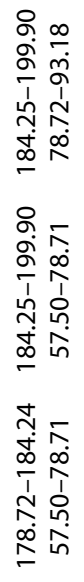 & 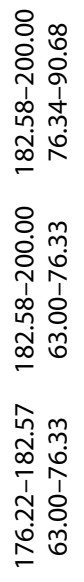 & 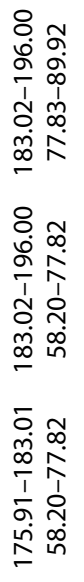 & 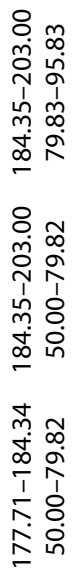 \\
\hline 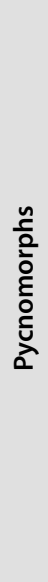 & $\equiv$ & 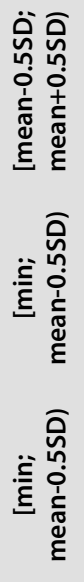 & 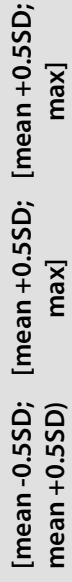 & 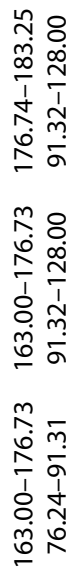 & 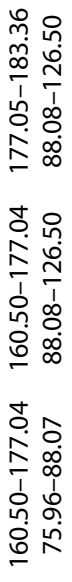 & 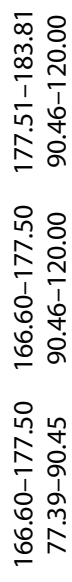 & 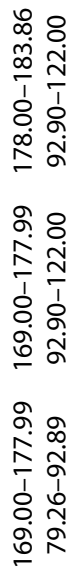 & 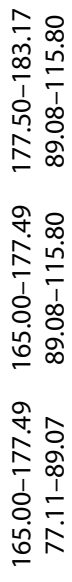 & 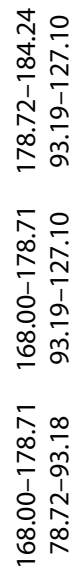 & 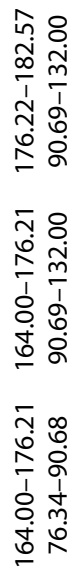 & 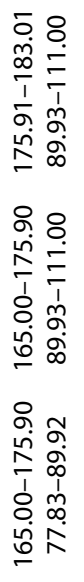 & 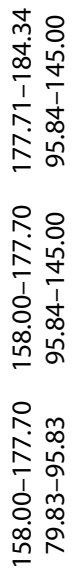 \\
\hline & ัํㅀ & 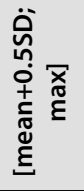 & 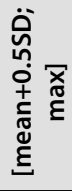 & 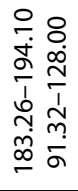 & 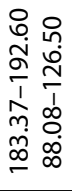 & 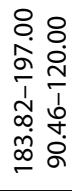 & 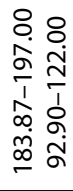 & 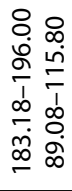 & 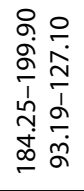 & 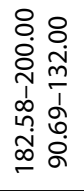 & 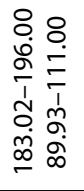 & 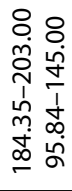 \\
\hline & $\frac{\xi}{\frac{\xi}{3}}$ & 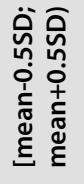 & 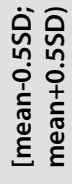 & 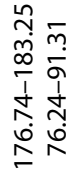 & 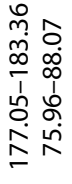 & 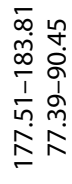 & 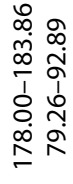 & 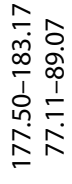 & 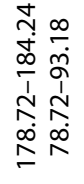 & 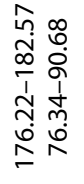 & 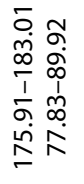 & 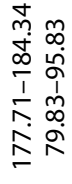 \\
\hline & $\begin{array}{l}\overline{\bar{\sigma}} \\
\text { ఓ్ }\end{array}$ & 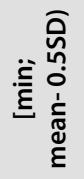 & 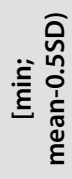 & 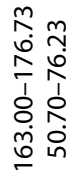 & 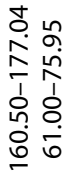 & 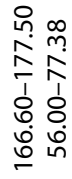 & 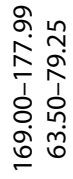 & 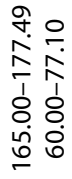 & 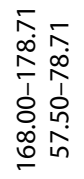 & 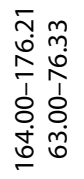 & 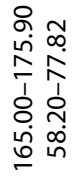 & 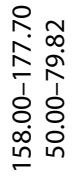 \\
\hline & gั & 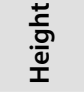 & $\begin{array}{l}\text {.0 } \\
\text { 30 }\end{array}$ & $\stackrel{\infty}{\sim}$ & จे & m & $\bar{m}$ & $\tilde{m}$ & $\stackrel{m}{m}$ & $\stackrel{\text { mे }}{ }$ & & \\
\hline
\end{tabular}




\begin{tabular}{|c|c|c|c|c|c|c|c|c|c|c|c|c|}
\hline 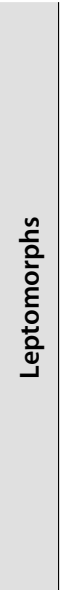 & $=$ & 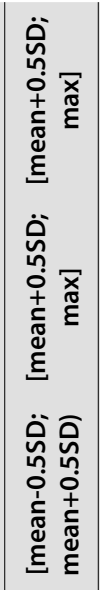 & 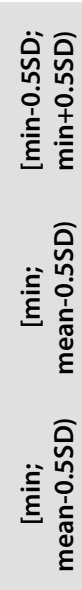 & 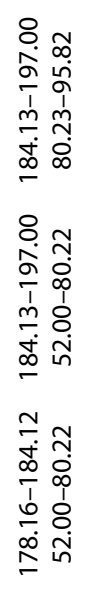 & 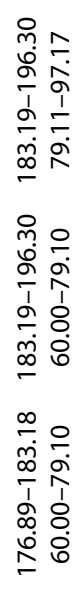 & 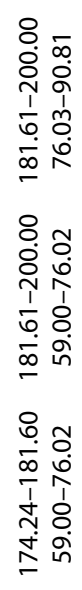 & 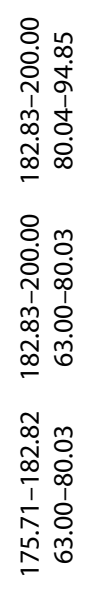 & 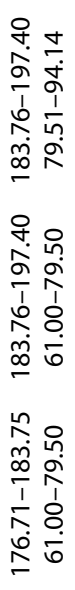 & 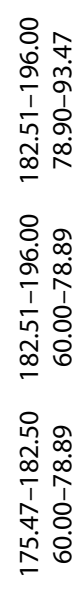 & 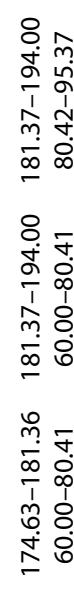 & 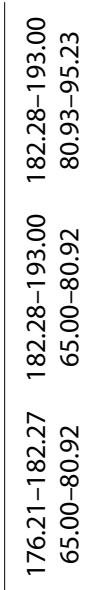 & \\
\hline 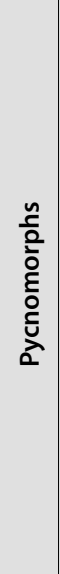 & $\equiv$ & 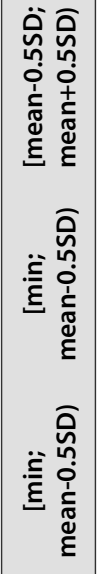 & 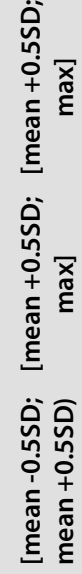 & 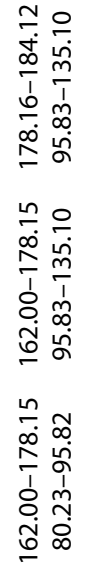 & 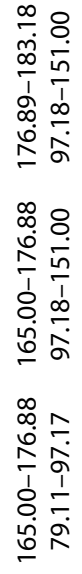 & 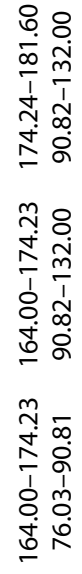 & 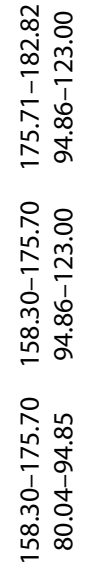 & 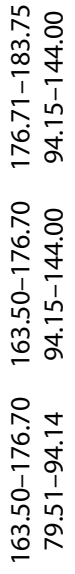 & 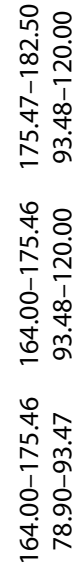 & 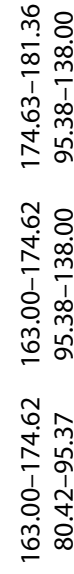 & 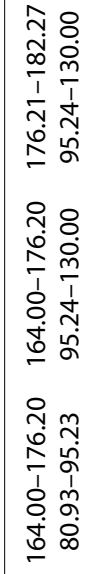 & \\
\hline & 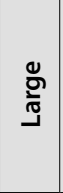 & 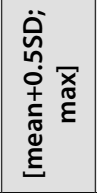 & 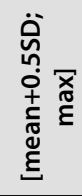 & 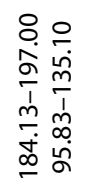 & 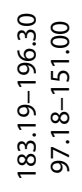 & 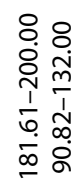 & 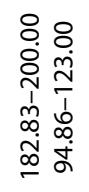 & 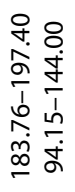 & 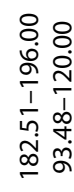 & $\begin{array}{l}8 \\
+ \\
\frac{j}{1} \\
\frac{1}{m} \\
\frac{\infty}{\infty} \\
\frac{1}{n}\end{array}$ & 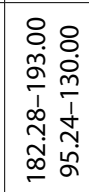 & \\
\hline & 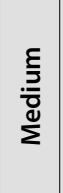 & 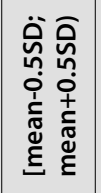 & 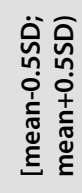 & 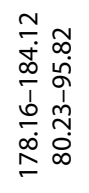 & 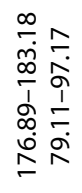 & 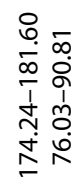 & 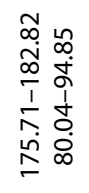 & 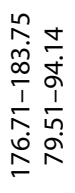 & 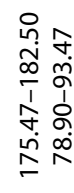 & 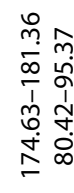 & 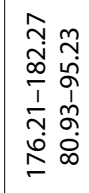 & \\
\hline & $\begin{array}{l}\overline{\bar{\sigma}} \\
\text { हn }\end{array}$ & 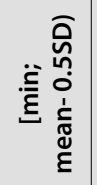 & 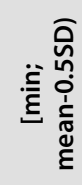 & 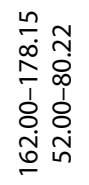 & 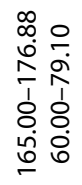 & 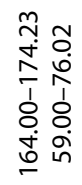 & 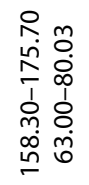 & 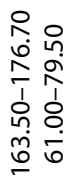 & 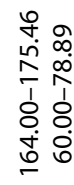 & 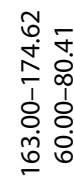 & 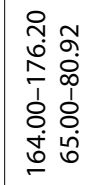 & \\
\hline & 离 & $\begin{array}{l}\text { 节 } \\
\frac{\sigma}{\mathbf{v}}\end{array}$ & & & & & f & & & & J & \\
\hline
\end{tabular}




\begin{tabular}{|c|c|c|c|c|c|c|c|c|c|c|c|c|}
\hline 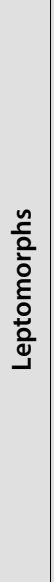 & $\equiv$ & 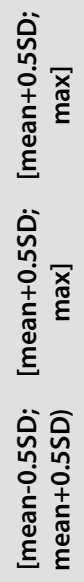 & 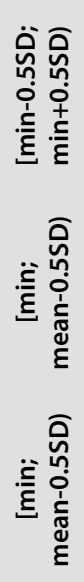 & 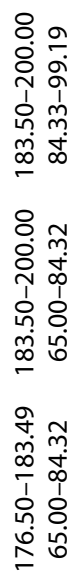 & 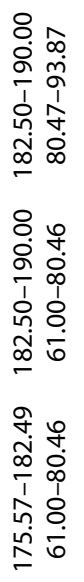 & 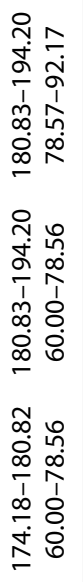 & 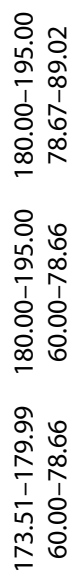 & 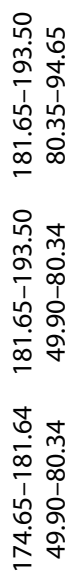 & 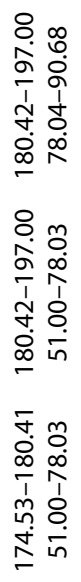 & 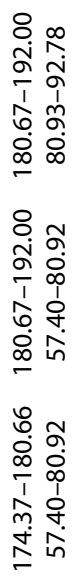 & 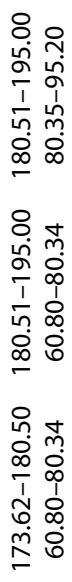 & 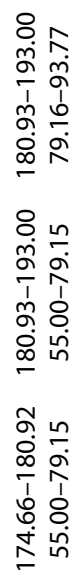 \\
\hline 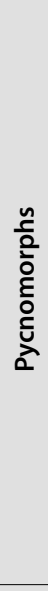 & $=$ & 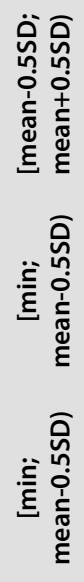 & 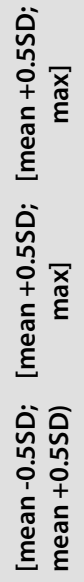 & 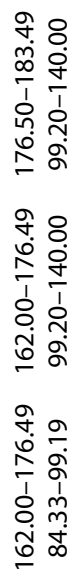 & 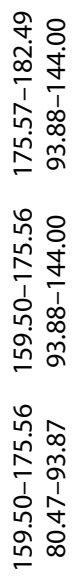 & 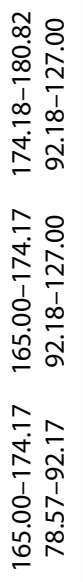 & 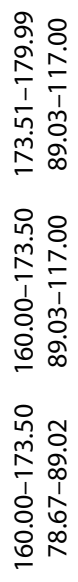 & 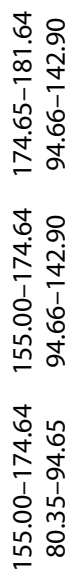 & 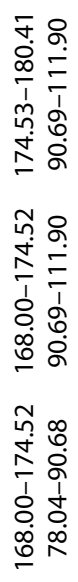 & 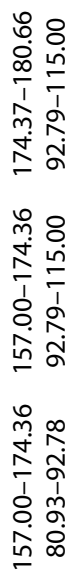 & 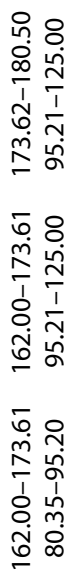 & 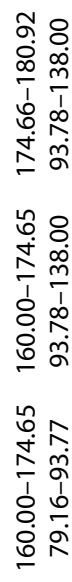 \\
\hline & פ্ & 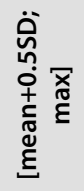 & 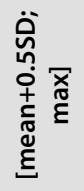 & 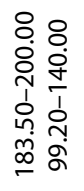 & 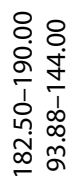 & 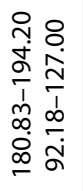 & 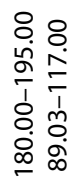 & 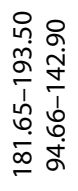 & 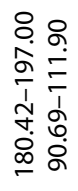 & 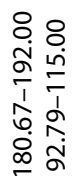 & 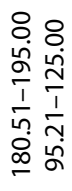 & 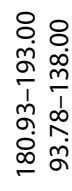 \\
\hline & $\frac{\xi}{\frac{\xi}{3}}$ & 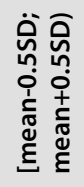 & 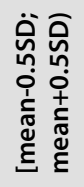 & 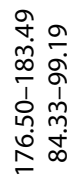 & 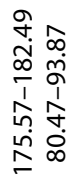 & 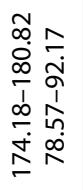 & 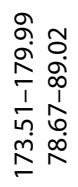 & 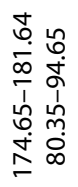 & 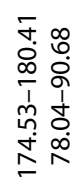 & 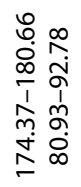 & 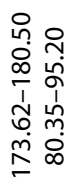 & 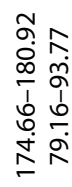 \\
\hline & $\begin{array}{l}\overline{\bar{\sigma}} \\
\stackrel{\text { ह }}{\text { ज }}\end{array}$ & 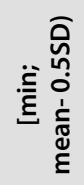 & 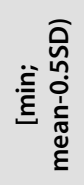 & 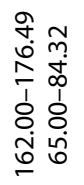 & 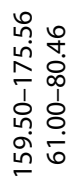 & 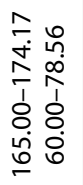 & 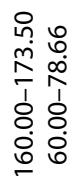 & 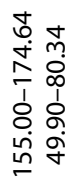 & 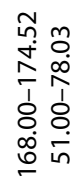 & 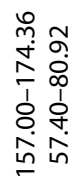 & 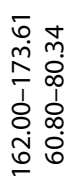 & 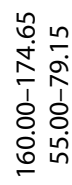 \\
\hline & 亗 & 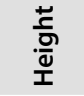 & $\frac{\text {.0 }}{3}$ & fo & $\hat{f}$ & $\stackrel{\infty}{+}$ & $\stackrel{q}{q}$ & in & in & กี & ณn & in \\
\hline
\end{tabular}




\begin{tabular}{|c|c|c|c|c|c|c|c|c|c|c|c|c|}
\hline $\begin{array}{l}\frac{n}{2} \\
\frac{0}{2} \\
\frac{0}{\xi} \\
0 \\
\frac{0}{0}\end{array}$ & $\equiv$ & 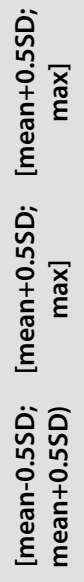 & 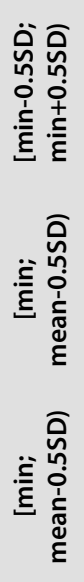 & 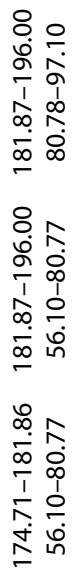 & 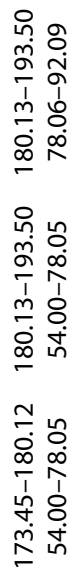 & 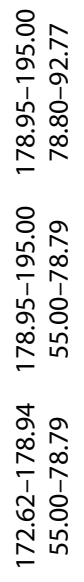 & 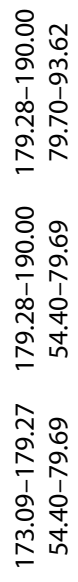 & 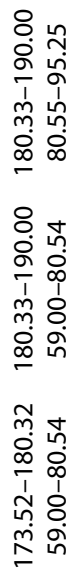 & 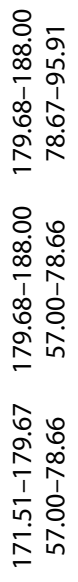 & 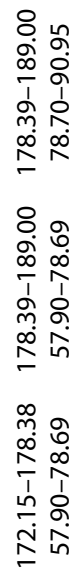 & 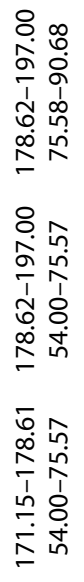 & 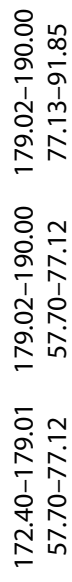 \\
\hline 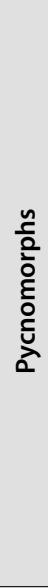 & $=$ & 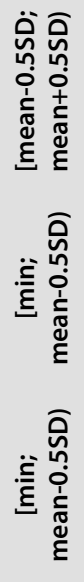 & 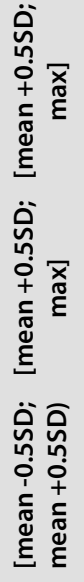 & 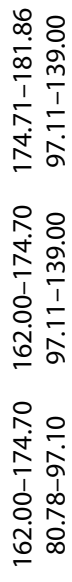 & 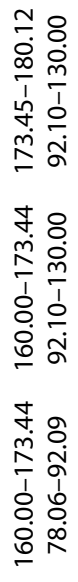 & 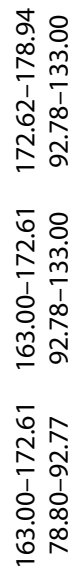 & 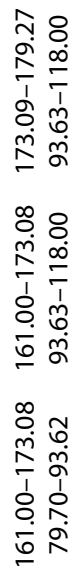 & 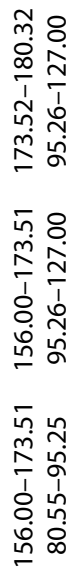 & 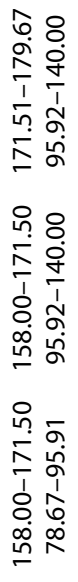 & 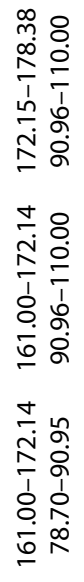 & 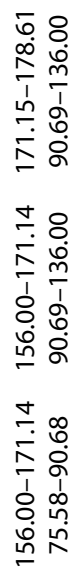 & 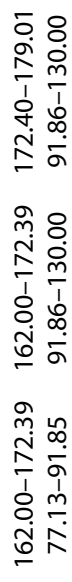 \\
\hline & 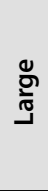 & 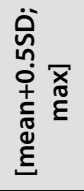 & 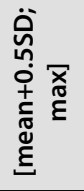 & 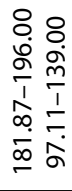 & 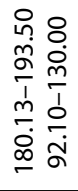 & 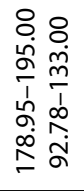 & 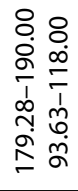 & 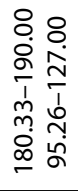 & 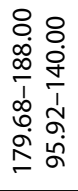 & 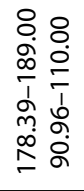 & 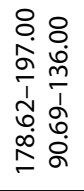 & 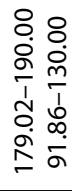 \\
\hline & $\begin{array}{l}\frac{\varepsilon}{3} \\
\frac{3}{d} \\
\sum\end{array}$ & 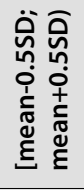 & 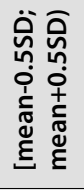 & 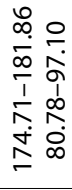 & 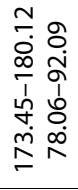 & 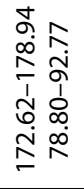 & 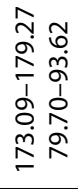 & 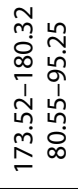 & 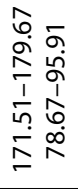 & 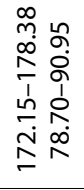 & 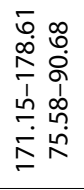 & 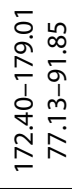 \\
\hline & $\begin{array}{l}\overline{\bar{\sigma}} \\
\text { है }\end{array}$ & 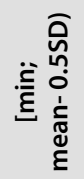 & 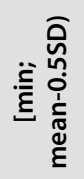 & 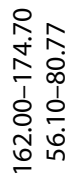 & 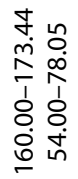 & 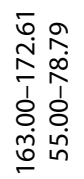 & 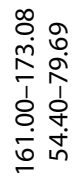 & 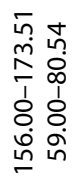 & 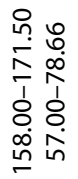 & 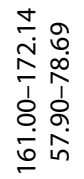 & 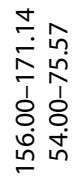 & 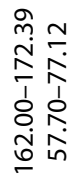 \\
\hline & 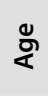 & 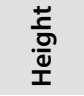 & $\frac{5}{\frac{0}{0}}$ & 织 & in & in & in & in & 응 & $\bar{\sigma}$ & ช్ & \\
\hline
\end{tabular}




\begin{tabular}{|c|c|c|c|c|c|c|c|c|c|c|}
\hline 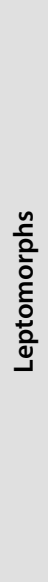 & - & 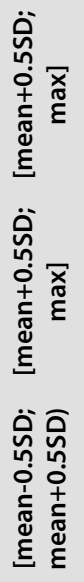 & 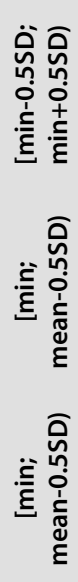 & 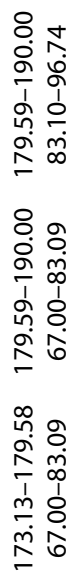 & 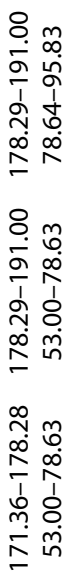 & 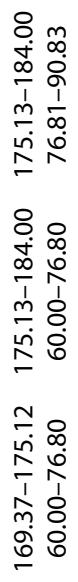 & 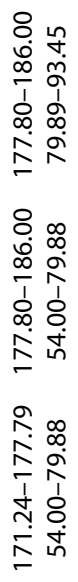 & 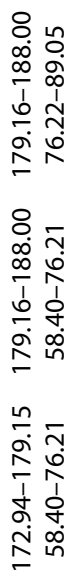 & 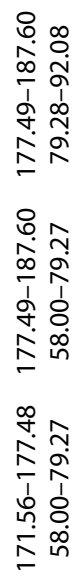 & 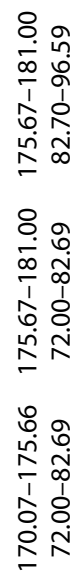 \\
\hline 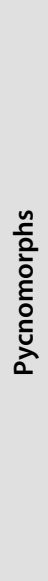 & $\equiv$ & 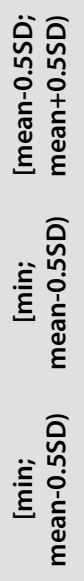 & 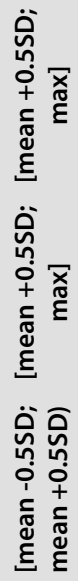 & 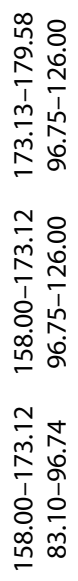 & 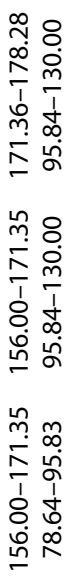 & 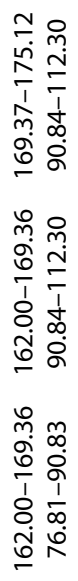 & 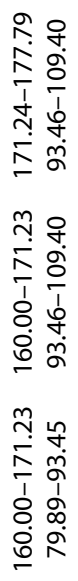 & 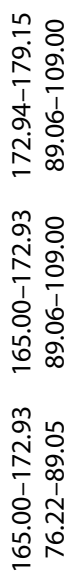 & 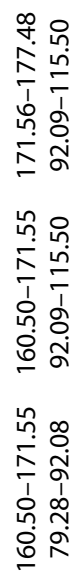 & 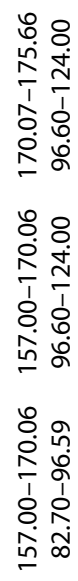 \\
\hline & ฮั & 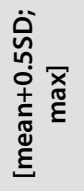 & 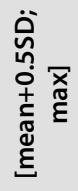 & 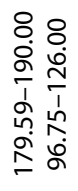 & 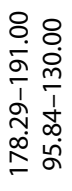 & 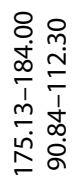 & 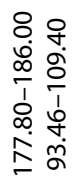 & 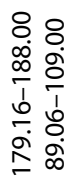 & 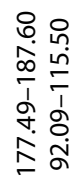 & 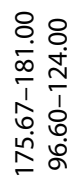 \\
\hline & $\begin{array}{l}\underline{\xi} \\
\frac{\xi}{\bar{z}} \\
\frac{0}{\Sigma}\end{array}$ & 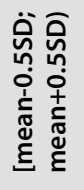 & 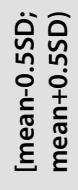 & 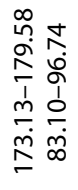 & 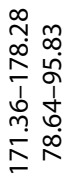 & 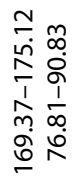 & 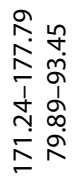 & 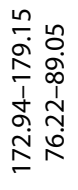 & 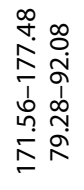 & 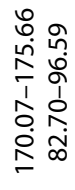 \\
\hline & 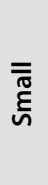 & 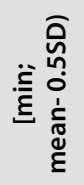 & 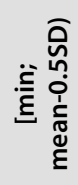 & 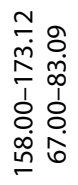 & 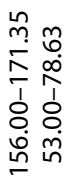 & 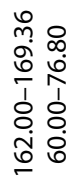 & 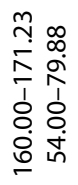 & 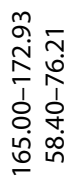 & 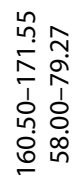 & 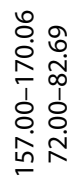 \\
\hline & 芩 & 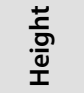 & 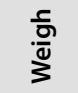 & t & กิ & 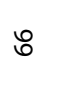 & 6 & 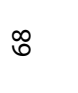 & 오 & \\
\hline
\end{tabular}




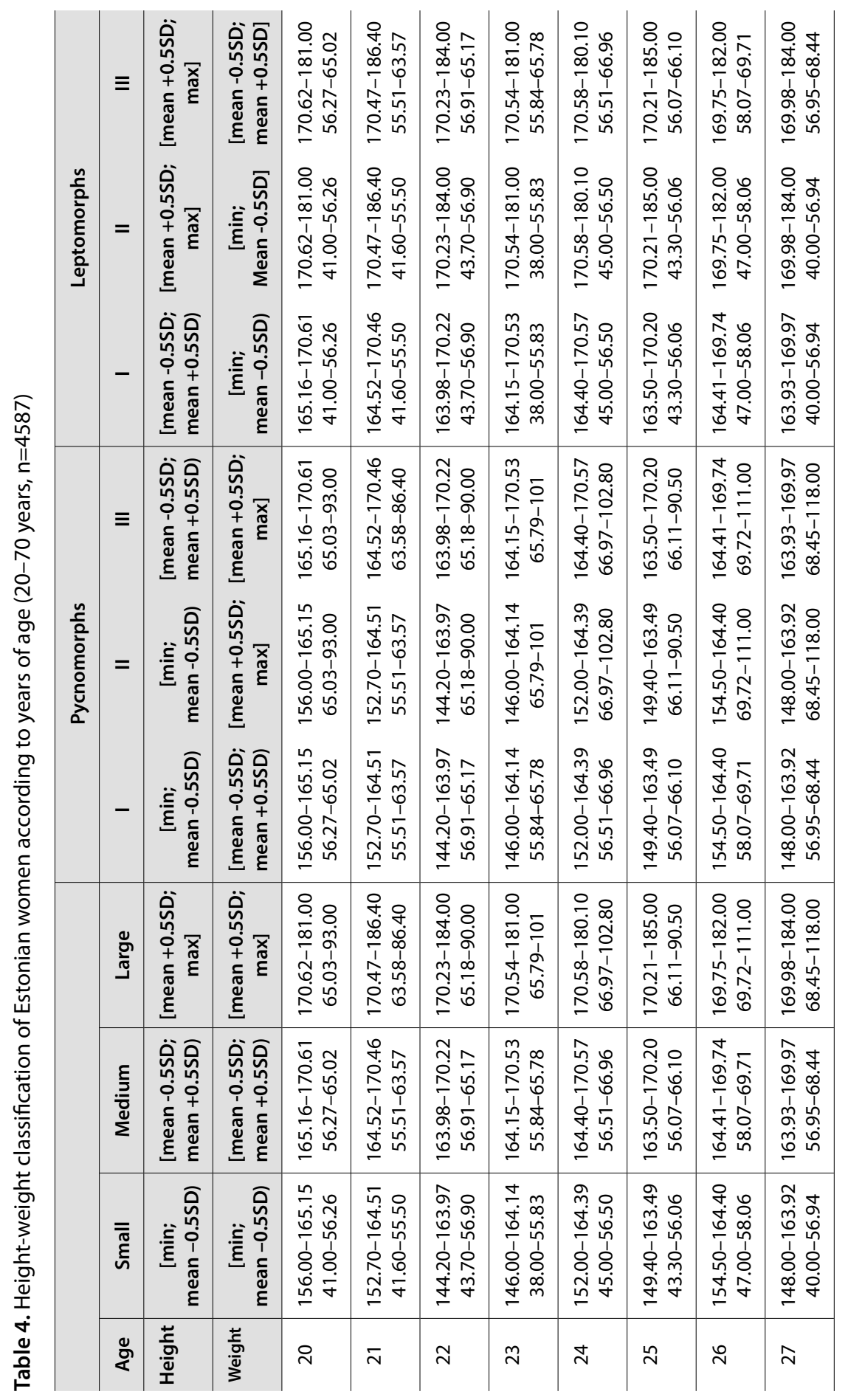




\begin{tabular}{|c|c|c|c|c|c|c|c|c|c|c|c|c|}
\hline 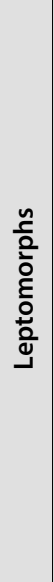 & $=$ & 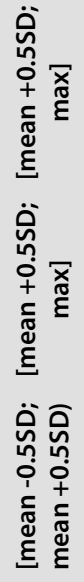 & 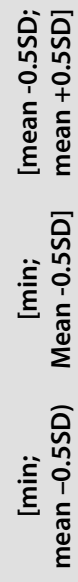 & 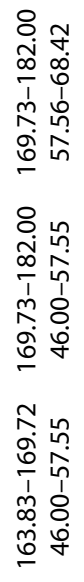 & 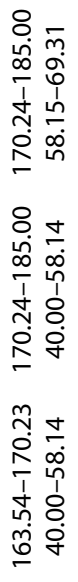 & 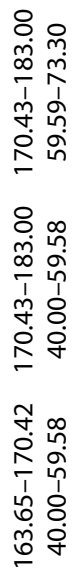 & 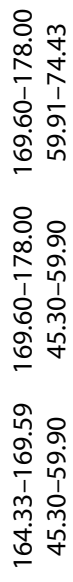 & 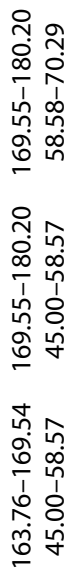 & 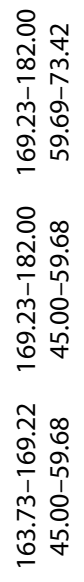 & 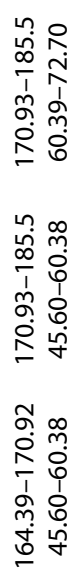 & 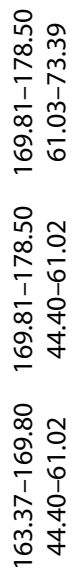 & 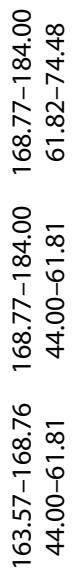 \\
\hline 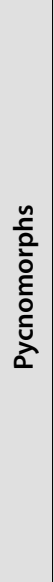 & $\equiv$ & 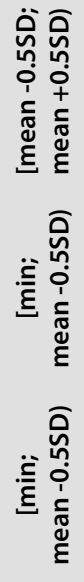 & 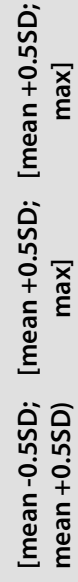 & 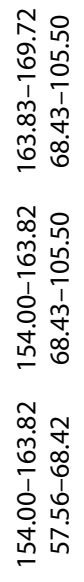 & 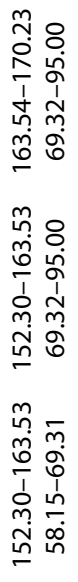 & 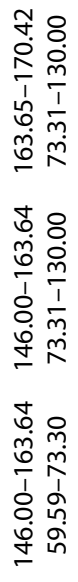 & 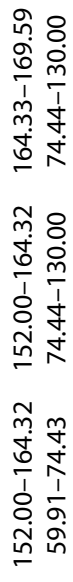 & 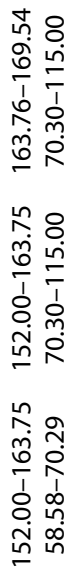 & 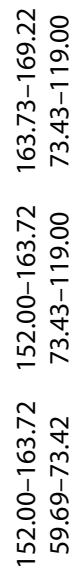 & 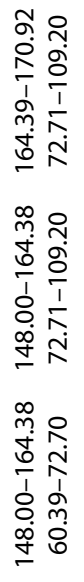 & 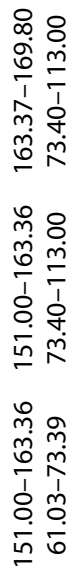 & 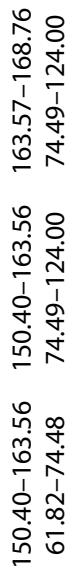 \\
\hline & 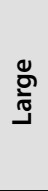 & 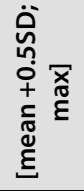 & 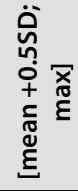 & 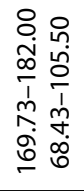 & 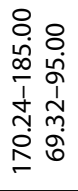 & 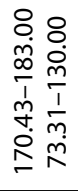 & 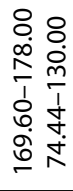 & 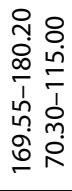 & 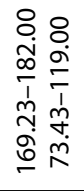 & 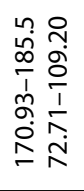 & 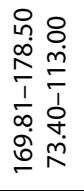 & 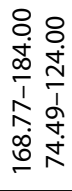 \\
\hline & $\frac{\xi}{\frac{\xi}{3}}$ & 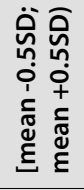 & 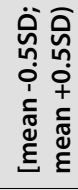 & 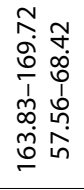 & 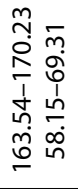 & 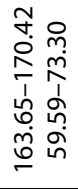 & 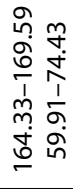 & 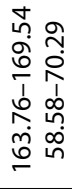 & 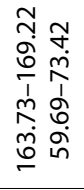 & 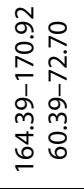 & 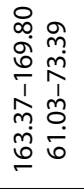 & 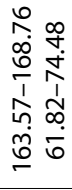 \\
\hline & $\begin{array}{l}\overline{\bar{\sigma}} \\
\text { ఓ్ }\end{array}$ & 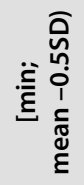 & 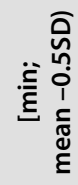 & 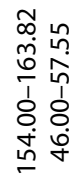 & 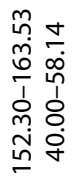 & 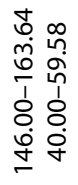 & 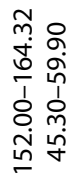 & 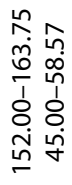 & 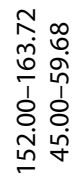 & 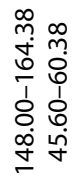 & 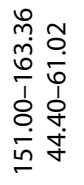 & 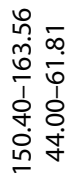 \\
\hline & 芩 & 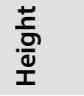 & 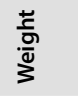 & $\stackrel{\infty}{\sim}$ & શิ & 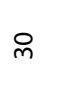 & $\bar{m}$ & $m$ & $\stackrel{m}{m}$ & $\stackrel{+}{m}$ & 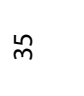 & \\
\hline
\end{tabular}




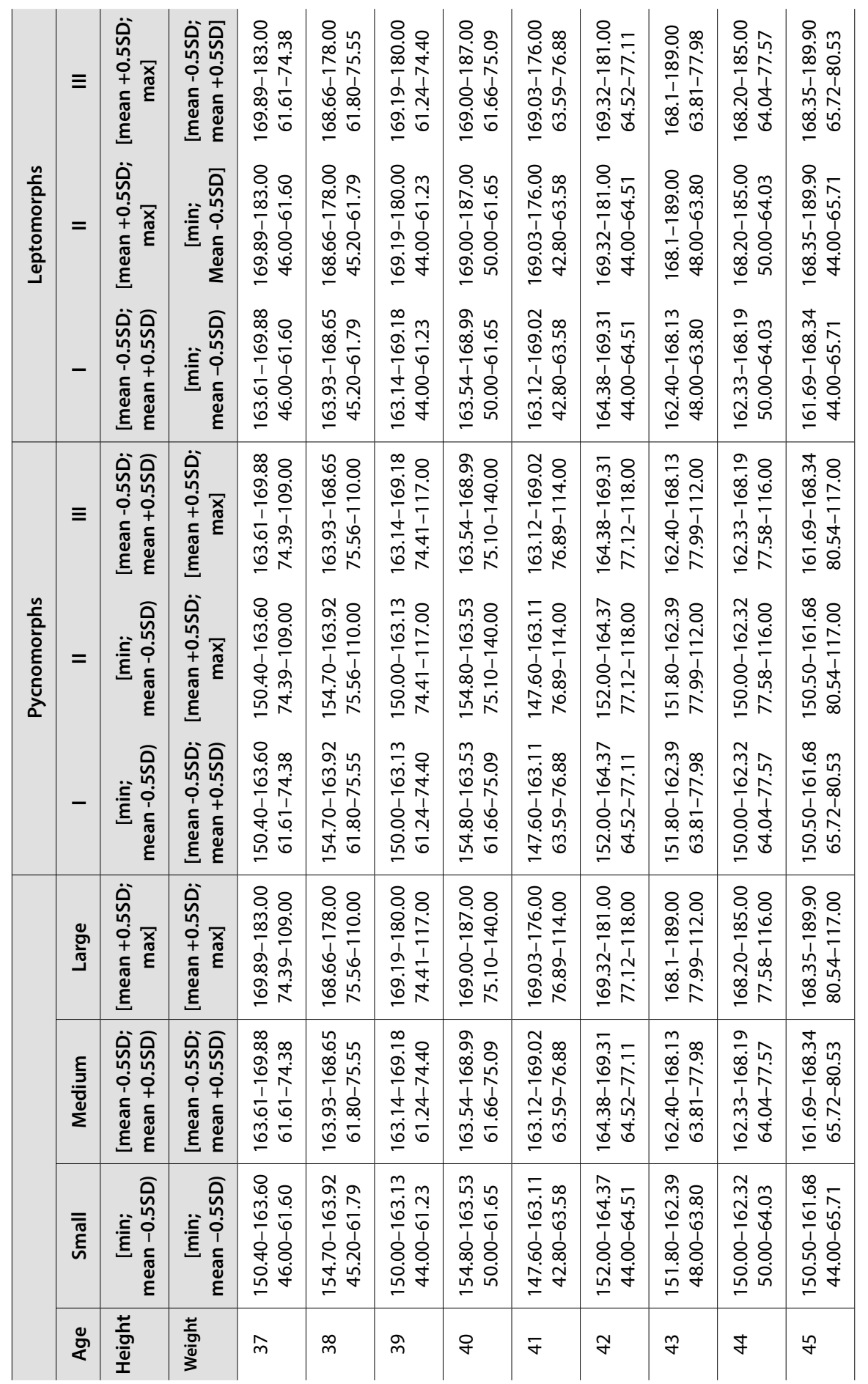




\begin{tabular}{|c|c|c|c|c|c|c|c|c|c|c|c|c|}
\hline 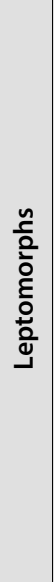 & $=$ & 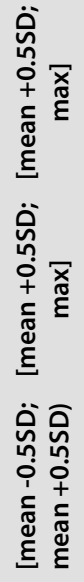 & 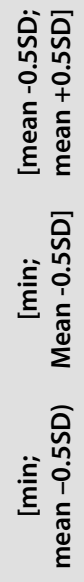 & 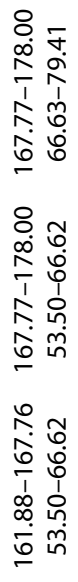 & 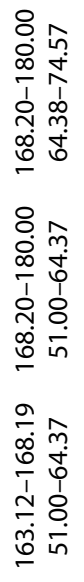 & 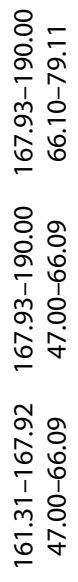 & 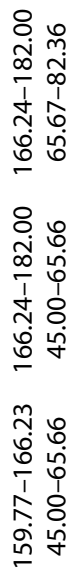 & 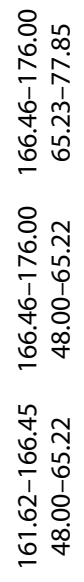 & 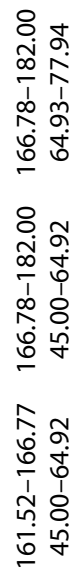 & 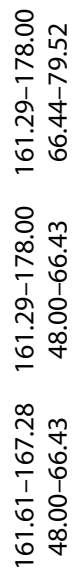 & 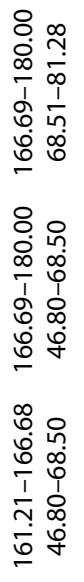 & 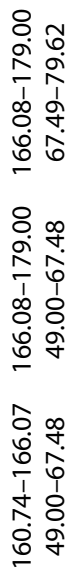 \\
\hline 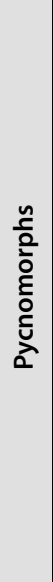 & $\equiv$ & 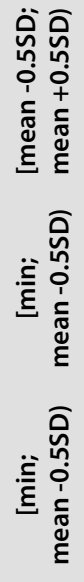 & 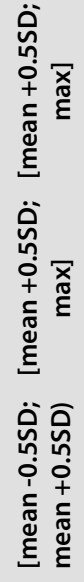 & 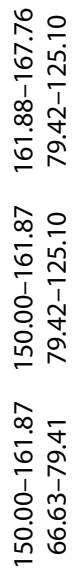 & 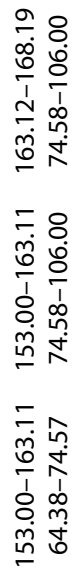 & 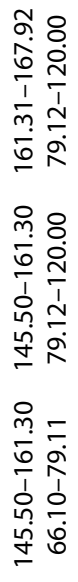 & 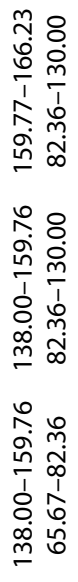 & 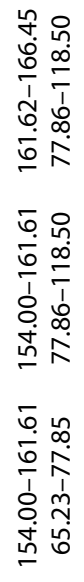 & 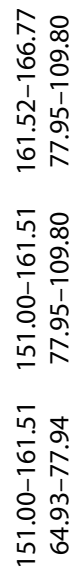 & 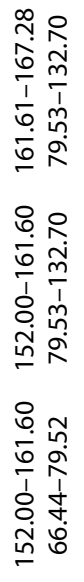 & 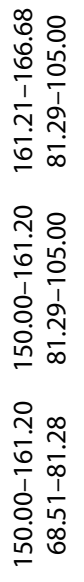 & 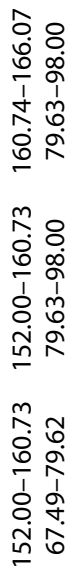 \\
\hline & 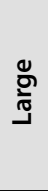 & 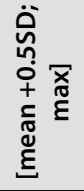 & 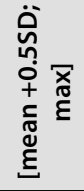 & 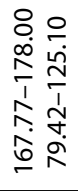 & 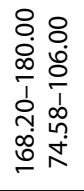 & 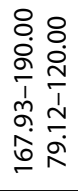 & 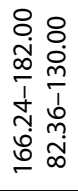 & 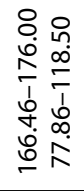 & 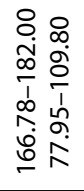 & 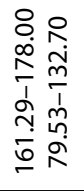 & 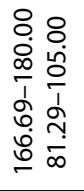 & 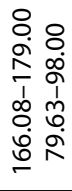 \\
\hline & $\frac{\xi}{\frac{\xi}{3}}$ & 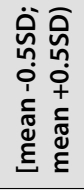 & 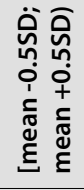 & 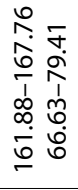 & 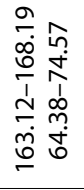 & 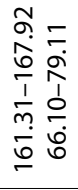 & 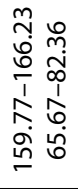 & 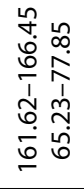 & 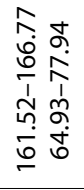 & 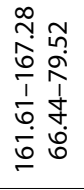 & 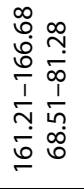 & 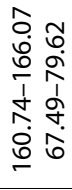 \\
\hline & $\begin{array}{l}\overline{\bar{\sigma}} \\
\text { ఓ్ }\end{array}$ & 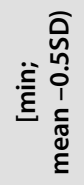 & 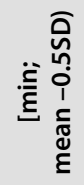 & 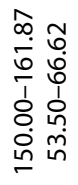 & 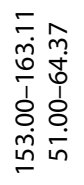 & 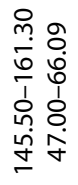 & 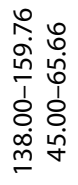 & 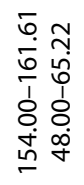 & 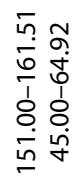 & 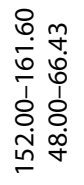 & 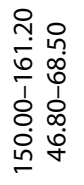 & 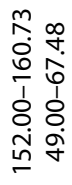 \\
\hline & 芩 & 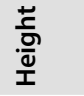 & 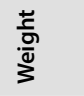 & $\mathscr{q}$ & $\sigma$ & $\stackrel{\infty}{q}$ & $g$ & 우 & in & กิ & & \\
\hline
\end{tabular}




\begin{tabular}{|c|c|c|c|c|c|c|c|c|c|c|c|c|}
\hline 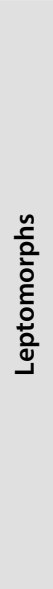 & $=$ & 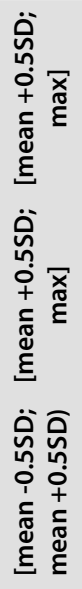 & 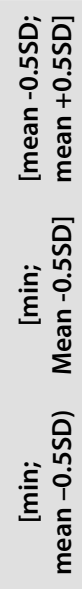 & 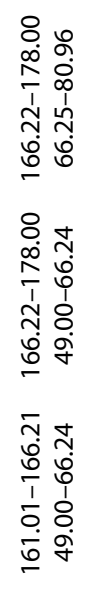 & 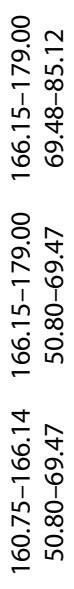 & 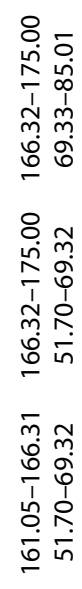 & 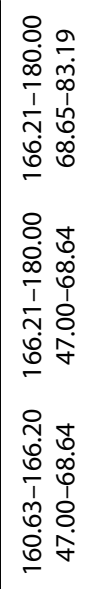 & 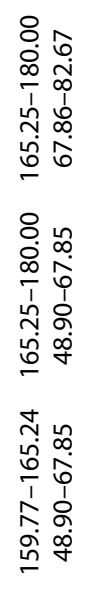 & 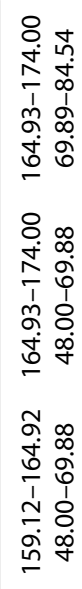 & 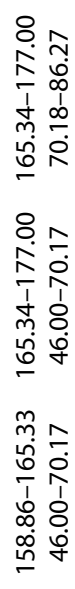 & 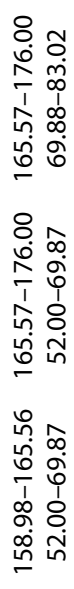 & 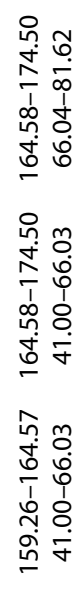 \\
\hline 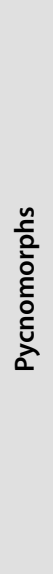 & $=$ & 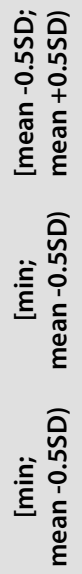 & 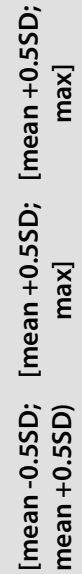 & 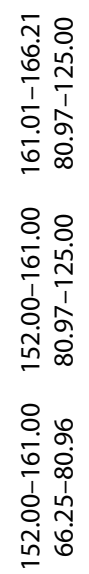 & 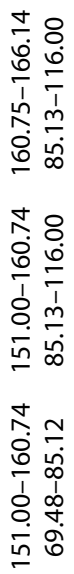 & 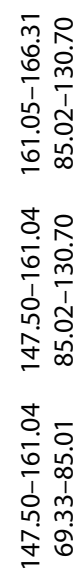 & 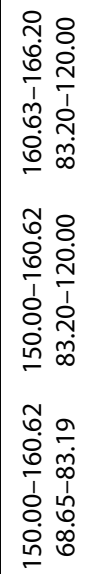 & 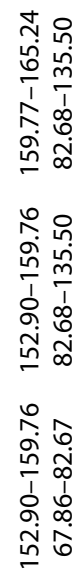 & 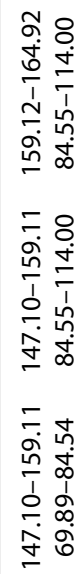 & 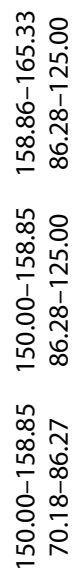 & 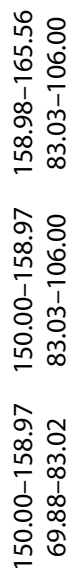 & 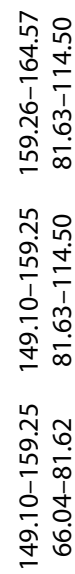 \\
\hline & 㞼 & 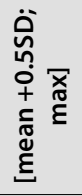 & 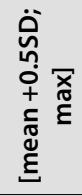 & 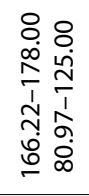 & 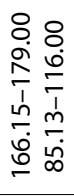 & 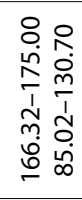 & 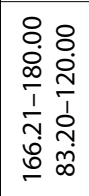 & 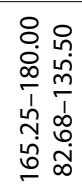 & 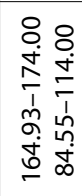 & 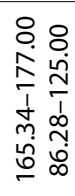 & 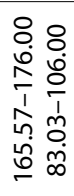 & 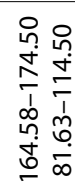 \\
\hline & 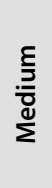 & 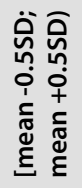 & 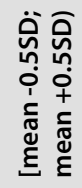 & 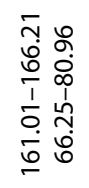 & 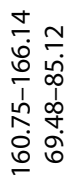 & 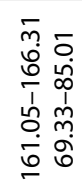 & 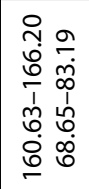 & 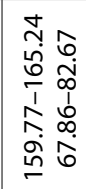 & 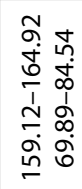 & 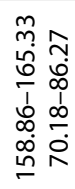 & 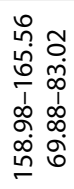 & \\
\hline & 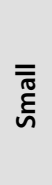 & 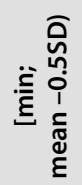 & 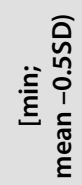 & 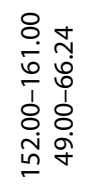 & 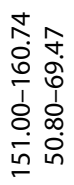 & 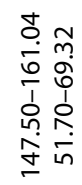 & 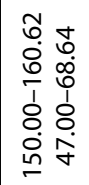 & 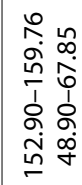 & 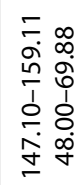 & 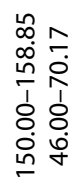 & 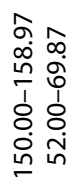 & \\
\hline & هัष & 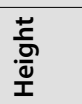 & 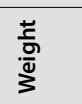 & in & in & in & $\stackrel{\infty}{n}$ & in & & $\overline{6}$ & ชิ & \\
\hline
\end{tabular}




\begin{tabular}{|c|c|c|c|c|c|c|c|c|c|c|}
\hline 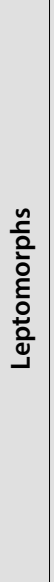 & $\equiv$ & 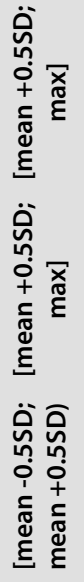 & 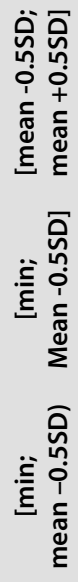 & 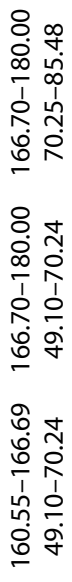 & 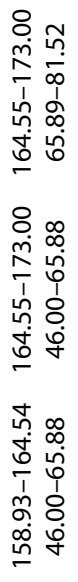 & 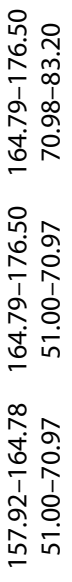 & 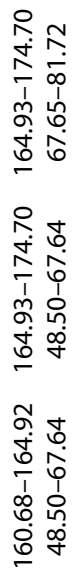 & 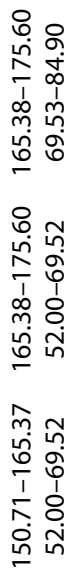 & 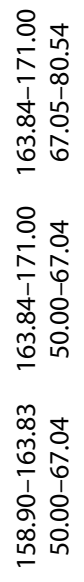 & 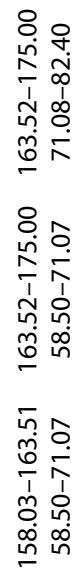 \\
\hline 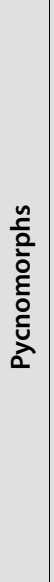 & $\equiv$ & 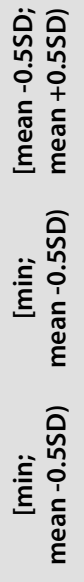 & 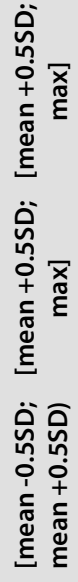 & 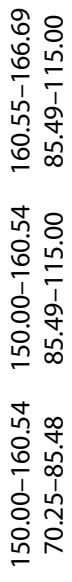 & 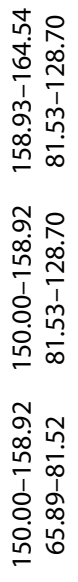 & 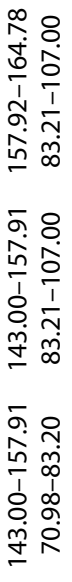 & 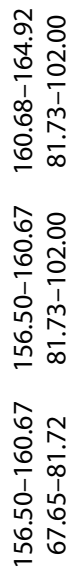 & 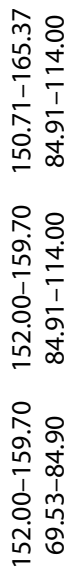 & 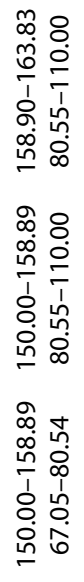 & 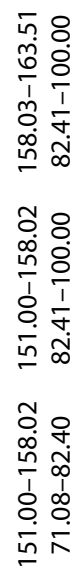 \\
\hline & 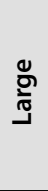 & 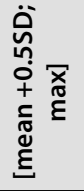 & 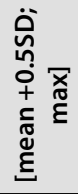 & 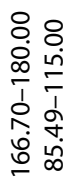 & 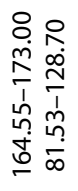 & 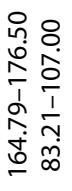 & 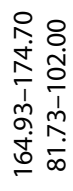 & 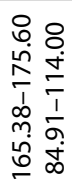 & 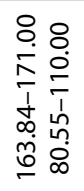 & 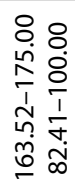 \\
\hline & 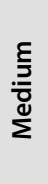 & 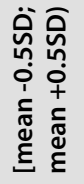 & 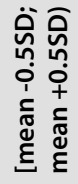 & 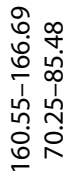 & 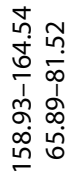 & 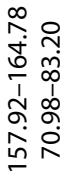 & 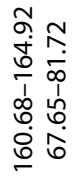 & 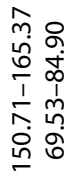 & 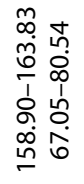 & 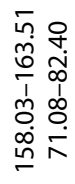 \\
\hline & $\begin{array}{l}\overline{\bar{\sigma}} \\
\text { है }\end{array}$ & 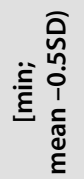 & 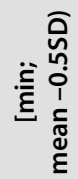 & 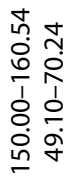 & 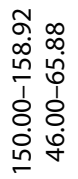 & 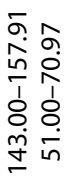 & 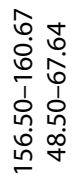 & 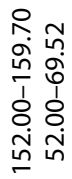 & 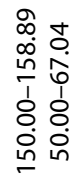 & 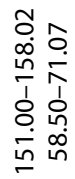 \\
\hline & 峁 & $\begin{array}{l}\frac{ \pm}{0} \\
\frac{0}{1} \\
\frac{1}{1}\end{array}$ & 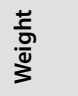 & $\mathbb{J}$ & $\tilde{\Omega}$ & 6 & $\widehat{0}$ & & & \\
\hline
\end{tabular}

